\title{
Statistical Analysis of Water Quality Indices from a Ramsar Site-Ropar Wetland, India
}

\author{
Saima Akhter*, Onkar Singh Brraich \\ Department of Zoology and Environmental Science, Punjabi University, India
}

Received January 26, 2020; Revised March 17, 2020; Accepted March 28, 2020

Copyright $\odot 2020$ by authors, all rights reserved. Authors agree that this article remains permanently open access under the terms of the Creative Commons Attribution License 4.0 International License

\begin{abstract}
By means of providing food and habitat for aquatic organisms including the threatened as well as endangered species, wetlands serve as earth's most important fresh water resource. As a good wetland is determined by its water quality, it is exigent to maintain the proper water quality of a wetland as the water quality of the fresh water resources is deteriorating at an alarming rate. Due to the contaminated water quality, human population also gets affected as they suffer from many waterborn diseases due to its usage, some of which may prove fatal. Therefore, it has become imperative to check the quality of water bodies at regular intervals for their sustainable management. In order to access the water quality, various physio-chemical parameters were carried out from October 2015 to September 2017 at four representative sites (S1, S2, S3 and S4). During the present study, it was found that various physico-chemical parameters greatly influence the water quality of the under study wetland. The average results of these parameters were compared with ISI, ICMR and WHO standards for drinking water quality. It could be inferred that water quality at Ropar wetland is "Poor" at S2 site and S4 site and "Very Poor" at S1 site and S3 site. DO and Free $\mathrm{CO}_{2}$ showed significant negative correlation with all other physico-chemical parameters at all the above mentioned sites.
\end{abstract}

Keywords Wetland, Pollution Status, Water Quality, Correlation

\section{Introduction}

Wetlands are major resource of fresh water and it was very essential part of human life, mainly for drinking and domestic purposes. The rapid increasing of human population and industrialization has created problems of disposal of waste water products. The undesirable and toxic substances are regularly discharged into the wetlands through surface run off that degrades the water quality (Lawson 2011). Water quality deals with the physical, chemical and biological characteristics in relation to all other hydrological properties. Product quality and safety is greatly reduced by the environmental impacts as the variation in various water quality parameters influences management decisions in aquaculture (Chhatawal, 1998). Current information about the concentration of various solutes at a given place and time is important which is provided by the quality of the water. Water quality provide the basis for judging the suitability of water for its designated use and in order to improve its existing conditions (Lloyd, 1992). The major concerns in terms of water quantity and quality is the unequal distribution of water on the surface of the earth and fast declining availability of useable fresh water (Boyd and Tucker, 1998). In order to determine the state of pollution in our rivers, a continuous monitoring of water quality is essential. Communication of this information to the general public and the Government is very important so that the policies for the conservation of the precious fresh water resources are developed (Ali et al., 2000). For the human and industrial growth, water is considered to be the main requirement. Increase in population and industrialization, the demand of the freshwater increases in the last decades. This demand fulfilled by the rivers which provide the water for human life and agriculture purposes. Due to the waste discharged from the human and industrial activities, the quality of river water has deteriorated which affects human as well as aquatic life. (Ramakrishnaiah et al., 2009; Jindal and Sharma, 2010). Substantial changes in the richness, composition and density of aquatic plant species in and around the lakes are caused by the degree of water quality degradation, particularly in terms of excess inputs of nutrients (Toivonen and Huttunen, 1995; Bini et al., 1999: Magee et al., 1999; Lougheed et al., 2001; Sisodia and Moundiotiya, 2006). Wetlands are fragile aquatic ecosystems. Slight change in the aquatic environment causes serious alterations in various abiotic and biotic 
components of the wetlands. Ropar wetland is designated as Ramsar site in 2002. Now, it is experiencing rampant deforestation in the catchment area which is ultimately discharging loads of silt into it. Small islands have been emerged in the reservoir area of this wetland which has drastically reduced its water carrying capacity as well as depth. The incoming of silt from adjoining areas is altering its abiotic characteristics which render the water unfit for the survival of fish as well as many other aquatic organisms. Pollution from the adjoining industries (National Fertilizer Limit (NFL) Nangal) is also one of the major causes of degradation of this wetland. Discharge of effluents, heavy metals and ash from the thermal plant and slit from cement plant causes mass mortality of fishes and poses a serious threat to other biotic components of this internationally important wetland. Thus, present study revealed that the extent of pollution in the river which is directly affecting the flora and fauna. Hence, this study suggests that regular bio-monitoring of this water body should be carried out to keep its flora and fauna intact for the years to come. The diversity of organisms found in this wetland gives a clear indication about the quality of water and habitat. Keeping in view of the above facts present study is endowed in this direction to survey and document the statistical analysis of water quality indices of the Ropar wetland.

\section{Materials and Methods}

\subsection{Study Area}

Ropar Wetland is a manmade freshwater riverine as well as lacustrine wetland. It came into existence with the impoundment of water by constructing a barrage on the river Sutlej near Ropar town. It is situated at $30^{\circ} 58^{\prime}-31^{\circ} 02^{\prime} \mathrm{N}$ latitude and $76^{\circ} 30^{\prime}-76^{\circ} 33^{\prime} \mathrm{E}$ longitude (Figure 1). This important ecological zone is located in the Shivalik foothills of the Lower Himalayas and was created in 1952 on the Sutlej River, in the Punjab State of India, by building a head regulator to store and divert water for beneficial uses of irrigation through canals, drinking and industrial water supply. Shallow water features exist along both the sides of the river located within the wetland area. Climatically this area is sub-moist or humid and comparatively less hot region of Punjab with mean annual rainfall of $1518 \mathrm{~mm}$. The total area covered by the Wetland is 1365 ha, which includes 800 ha area of the river and the reservoir, 30 ha of forest area named as Sadabarat Forest and 30 ha under marshy plants, which serves as an important habitat for some rare and threatened species of Shivalik foothills. It is also an important staging and resting ground for migratory waterfowl (Ladhar, 2005).

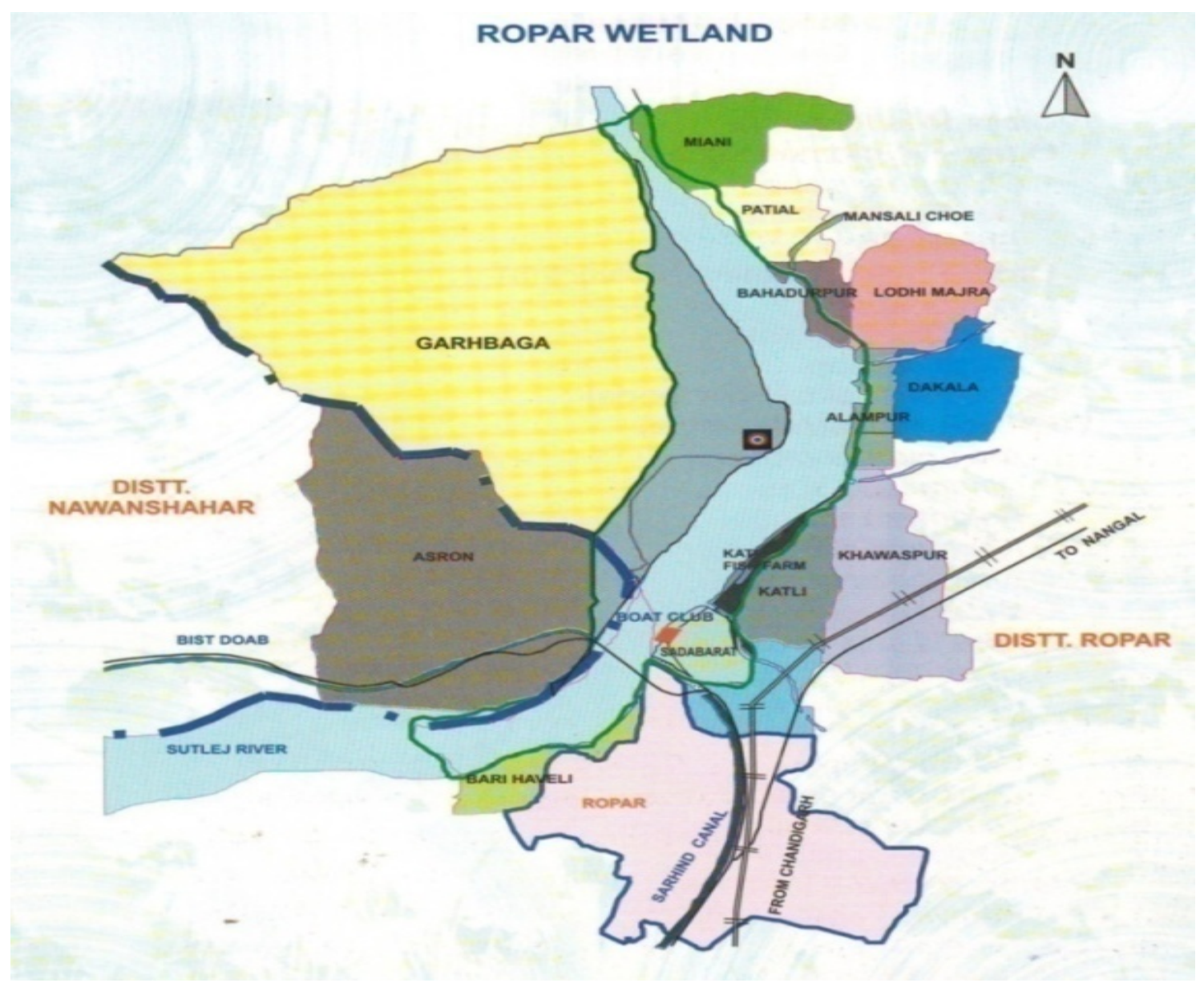

Figure 1. Map of the study area (Ropar Wetland) 


\subsection{Physico-Chemical Analysis}

Water samples for physico-chemical analysis from the different spots have been collected monthly from October, 2015 to September, 2017. Water samples were collected in 2 liters polythene bottles and the exact sample location was fixed by Global Positioning System (GPS). Before sampling, the bottles were washed thoroughly with detergent, tap water and then distilled water in order to remove the impurities from the bottle. Physico-chemical parameters like air and water temperature, conductivity, total dissolved solid, total alkalinity, salinity, $\mathrm{pH}$, free carbon dioxide and dissolved oxygen have been analyzed on the spot with the help of portable water analysis kit as their values are liable to change soon. The rest of the parameters viz., turbidity, total hardness, calcium and magnesium hardness, chlorides, nitrites, nitrates, phosphates and sulphates were analyzed in the laboratory by titrimetric methods using standard methodology (Trivedi and Goel, 1984; APHA, 2012) within the following 2 to 3 days during which samples were kept in cold storage. The results obtained were compared with standard values prescribed by World Health Organisation (WHO, 1992), Indian Standard Institute (ISI, 1973) and Indian Council of Medical Research (ICMR, 1975).

\subsection{Water Quality Index (WQI) Computation}

The concept of indices to represent gradation in water quality was first proposed by Horton (1965). It indicates the quality by an index number, which represents the overall quality of water for any intended use. WQI is commonly used for the detection and evaluation of water pollution and may be defined as "a rating reflecting the composite influence of different quality parameters on the overall quality of water" (Deininger and Maciunas, 1971; Harkins, 1974; Mishra and Patel, 2005). Ten parameters namely TDS, pH, Alkalinity, Dissolved oxygen, Salinity, Chlorides, Total Hardness, $\mathrm{Ca}++$ Hardness, $\mathrm{Mg}++$ Hardness and sulphates were employed to calculate WQI.
Weighted arithmetic water quality index method classified the water quality according to the degree of purity by using the most commonly measured water quality variables. The method has been widely used by various scientists and the calculation of WQI was made by using the following equation (Brown et al., 1972):

$$
\mathrm{WQI}=\Sigma \mathrm{Q}_{\mathrm{i}} \mathrm{W}_{\mathrm{i}} / \Sigma \mathrm{W}_{\mathrm{i}}
$$

The quality rating scale (Qi) for each parameter is calculated by using this expression

$$
\mathrm{Q}_{\mathrm{i}}=100\left[\left(\mathrm{~V}_{\mathrm{i}}-\mathrm{V}_{\mathrm{o}} / \mathrm{S}_{\mathrm{i}}-\mathrm{V}_{\mathrm{o}}\right)\right]
$$

Where, $V_{i}$ is estimated concentration of ith parameter in the analyzed water $\mathrm{V}_{\mathrm{o}}$ is the ideal value of this parameter in pure water $\mathrm{V}_{\mathrm{o}}=0$ (except $\mathrm{pH}=7.0$ and $\left.\mathrm{DO}=14.6 \mathrm{mg} / \mathrm{l}\right), \mathrm{S}_{\mathrm{i}}$ is the recommended standard value of ith parameter. The unit weight $\left(\mathrm{W}_{\mathrm{i}}\right)$ for each water quality parameter is calculated by using the following formula:

$$
\mathrm{Wi}=\mathrm{K} / \mathrm{Si}
$$

Where, $\mathrm{K}=$ proportionality constant and can also be calculated by using the following equation:

$$
\mathrm{K}=1 / \Sigma\left(1 / \mathrm{S}_{\mathrm{i}}\right)
$$

\section{Results}

\subsection{Analysis of Physico-Chemical Parameters}

The study on Ropar wetland was carried out from October 2015 to September 2017 to assess the water quality at four representative sites (S1, S2, S3 and S4). The correlation between different physico-chemical parameters were also analyzed statistically by using Pearson correlation analysis at all sites (S1, S2, S3 and S4) and was depicted in Table 7,8,9,10. During the present investigations water qualities of Ropar wetland was

\begin{tabular}{|c|c|c|c|c|c|}
\hline Parameters & Observed Value $\left(\mathrm{V}_{\mathrm{i}}\right)$ & Standard Value $\left(\mathrm{S}_{\mathrm{i}}\right)$ & Unit Weight $\left(\mathrm{W}_{\mathrm{i}}\right)$ & Quality Rating $\left(\mathbf{Q}_{\mathrm{i}}\right)$ & $\mathbf{W}_{\mathrm{i}} \mathbf{Q}_{\mathrm{i}}$ \\
\hline TDS(mg/l) & 129.62 & 500 & 0.005 & 25.92 & 0.129 \\
\hline Dissolved oxygen(mg/l) & 7.66 & 5 & 0.503 & 72.29 & 36.36 \\
\hline pH* & 7.65 & $6.5-8.5$ & 0.335 & 15 & 5.025 \\
\hline Alkalinity(mg/l) & 105.66 & 120 & 0.02 & 88.05 & 1.76 \\
\hline Salinity(mg/l) & 173.16 & 500 & 0.005 & 34.63 & 0.173 \\
\hline Chlorides(mg/l) & 25.09 & 250 & 0.01 & 10.03 & 0.1 \\
\hline Total Hardness(mg/l) & 242.87 & 500 & 0.005 & 48.57 & 0.242 \\
\hline $\mathrm{Ca}^{++}$Hardness(mg/l) & 29.33 & 75 & 0.033 & 39.1 & 1.29 \\
\hline $\mathrm{Mg}^{++}$Hardness(mg/l) & 117.35 & 30 & 0.083 & 391.16 & 32.46 \\
\hline \multirow[t]{2}{*}{ Sulphates(mg/l) } & 2.96 & 500 & 0.005 & 0.59 & 0.002 \\
\hline & & & $\sum_{w_{i}}=1.004$ & & $\sum_{\mathrm{wiQi}}=77.23$ \\
\hline
\end{tabular}
analyzed by studying various physico-chemical parameters at all the sites and are represented in Table 1,2,3,4.

Table 1. Water quality rating and water quality index at S1 site of Ropar Wetland

All parameters are expressed in $\mathrm{mg} / \mathrm{l}$ except $\mathrm{pH}^{*}$ 
Table 2. Water quality rating and water quality index at S2 site of Ropar Wetland

\begin{tabular}{|c|c|c|c|c|c|}
\hline Parameters & Observed Value $\left(\mathrm{V}_{\mathrm{i}}\right)$ & Standard Value $\left(\mathrm{S}_{\mathrm{i}}\right)$ & Unit Weight $\left(\mathbf{W}_{\mathrm{i}}\right)$ & Quality Rating $\left(\mathbf{Q}_{\mathrm{i}}\right)$ & $\mathbf{W}_{\mathrm{i}} \mathbf{Q}_{\mathrm{i}}$ \\
\hline TDS(mg/l) & 124.2 & 500 & 0.005 & 24.84 & 0.124 \\
\hline Dissolved oxygen(mg/l) & 7.67 & 5 & 0.503 & 72.18 & 36.307 \\
\hline pH* & 7.23 & $6.5-8.5$ & 0.335 & 7 & 2.345 \\
\hline Alkalinity(mg/l) & 92.75 & 120 & 0.02 & 77.29 & 1.546 \\
\hline Salinity(mg/l) & 166.04 & 500 & 0.005 & 33.2 & 0.166 \\
\hline Chlorides(mg/l) & 23.13 & 250 & 0.01 & 9.25 & 0.925 \\
\hline Total Hardness(mg/l) & 247.45 & 500 & 0.005 & 49.49 & 0.247 \\
\hline $\mathrm{Ca}^{++}$Hardness(mg/l) & 26.59 & 75 & 0.033 & 35.45 & 1.17 \\
\hline $\mathbf{M g}^{++}$Hardness(mg/l) & 113.45 & 30 & 0.083 & 378.17 & 31.39 \\
\hline \multirow[t]{2}{*}{ Sulphates(mg/l) } & 2.4 & 500 & 0.005 & 0.5 & 0.0025 \\
\hline & & & $\sum_{w_{i}}=1.004$ & & $\sum_{\mathrm{wiQi}}=74.22$ \\
\hline
\end{tabular}

All parameters are expressed in $\mathrm{mg} / \mathrm{l}$ except $\mathrm{pH}^{*}$

Table 3. Water quality rating and water quality index at S3 site of Ropar Wetland

\begin{tabular}{|c|c|c|c|c|c|}
\hline Parameters & Observed Value $\left(\mathrm{V}_{\mathrm{i}}\right)$ & Standard Value $\left(\mathrm{S}_{\mathrm{i}}\right)$ & Unit Weight $\left(\mathrm{W}_{\mathrm{i}}\right)$ & Quality Rating $\left(\mathbf{Q}_{\mathrm{i}}\right)$ & $\mathbf{W}_{\mathrm{i}} \mathbf{Q}_{\mathrm{i}}$ \\
\hline TDS(mg/l) & 130.7 & 500 & 0.005 & 26.14 & 0.001 \\
\hline Dissolved oxygen(mg/l) & 7.94 & 5 & 0.503 & 69.37 & 34.89 \\
\hline pH* & 7.66 & $6.5-8.5$ & 0.335 & 16 & 5.36 \\
\hline Alkalinity(mg/l) & 107.54 & 120 & 0.02 & 89.61 & 1.792 \\
\hline Salinity(mg/l) & 166.83 & 500 & 0.005 & 33.36 & 0.166 \\
\hline Chlorides(mg/l) & 25.33 & 250 & 0.01 & 10.13 & 0.101 \\
\hline Total Hardness(mg/l) & 248.66 & 500 & 0.005 & 49.73 & 0.248 \\
\hline $\mathrm{Ca}^{++}$Hardness(mg/l) & 28.9 & 75 & 0.033 & 38.53 & 1.271 \\
\hline $\mathrm{Mg}^{++}$Hardness(mg/l) & 118.01 & 30 & 0.083 & 393.36 & 32.64 \\
\hline \multirow[t]{2}{*}{ Sulphates(mg/l) } & 3.13 & 500 & 0.005 & 0.62 & 0.003 \\
\hline & & & $\sum_{w_{i}}=1.004$ & & $\sum_{\mathrm{WiQi}}=76.47$ \\
\hline
\end{tabular}

All parameters are expressed in $\mathrm{mg} / \mathrm{l}$ except $\mathrm{pH}^{*}$

Table 4. Water quality rating and water quality index at S4 site of Ropar Wetland

\begin{tabular}{|c|c|c|c|c|c|}
\hline Parameters & Observed Value $\left(\mathrm{V}_{\mathrm{i}}\right)$ & Standard Value $\left(\mathrm{S}_{\mathrm{i}}\right)$ & Unit Weight $\left(\mathrm{W}_{\mathrm{i}}\right)$ & Quality Rating ( $\left.\mathbf{Q}_{\mathbf{i}}\right)$ & $\mathbf{W}_{\mathrm{i}} \mathbf{Q}_{\mathrm{i}}$ \\
\hline TDS(mg/l) & 117.41 & 500 & 0.005 & 23.48 & 0.001 \\
\hline Dissolved oxygen(mg/l) & 7.48 & 5 & 0.503 & 74.16 & 37.3 \\
\hline pH* & 7.58 & $6.5-8.5$ & 0.335 & 8 & 2.68 \\
\hline Alkalinity(mg/l) & 90.95 & 120 & 0.02 & 75.79 & 1.515 \\
\hline Salinity(mg/l) & 154.58 & 500 & 0.005 & 30.91 & 0.154 \\
\hline Chlorides(mg/l) & 24.34 & 250 & 0.01 & 9.73 & 0.097 \\
\hline Total Hardness(mg/l) & 235.7 & 500 & 0.005 & 47.14 & 0.235 \\
\hline $\mathrm{Ca}^{++}$Hardness(mg/l) & 26.04 & 75 & 0.033 & 34.72 & 1.145 \\
\hline $\mathbf{M g}^{++}$Hardness(mg/l) & 110.3 & 30 & 0.083 & 367.66 & 30.51 \\
\hline \multirow[t]{2}{*}{ Sulphates(mg/l) } & 2.38 & 500 & 0.005 & 0.47 & 0.002 \\
\hline & & & $\sum \mathrm{wi}_{\mathrm{i}}=\mathbf{1 . 0 0 4}$ & & $\sum_{\mathrm{wiQi}}=73.64$ \\
\hline
\end{tabular}

All parameters are expressed in $\mathrm{mg} / \mathrm{l}$ except $\mathrm{pH}^{*}$ 
Table 5. Standards for drinking water

\begin{tabular}{|c|c|c|c|}
\hline Parameters & $\begin{array}{c}\text { WHO } \\
1992\end{array}$ & $\begin{array}{c}\text { ISI } \\
1973\end{array}$ & $\begin{array}{c}\text { ICMR } \\
1975 \\
\end{array}$ \\
\hline Conductivity $(\mu \mathrm{S} / \mathrm{cm})$ & 600 & - & - \\
\hline TDS(mg/l) & 500 & 500 & 500 \\
\hline Turbidity(NTU) & - & 10 & - \\
\hline Dissolved oxygen(mg/l) & 5 & - & 5 \\
\hline pH & $6.5-8.5$ & $6.5-8.5$ & 7.74 \\
\hline Alkalinity(mg/l) & 120 & 200 & 120 \\
\hline Salinity(mg/l) & 500 & - & - \\
\hline Chlorides(mg/l) & 250 & 250 & 250 \\
\hline Total Hardness(mg/l) & 500 & 300 & 200 \\
\hline $\mathrm{Ca}^{++}$Hardness(mg/l) & 75 & 75 & - \\
\hline $\mathrm{Mg}^{++}$Hardness(mg/l) & 30 & 30 & - \\
\hline Phosphates(mg/l) & 1 & - & - \\
\hline Sulphates(mg/l) & 500 & - & - \\
\hline Nitrates(mg/l) & 10 & - & - \\
\hline
\end{tabular}

Table 6. Water quality on the basis of WQI (Chatterji and Raziuddin, 2002)

\begin{tabular}{|c|c|}
\hline WQI LEVEL & Water Quality Rating \\
\hline $0-25$ & Excellent \\
\hline $26-50$ & Good \\
\hline $51-75$ & Poor \\
\hline $76-100$ & Very Poor \\
\hline$>100$ & Unfit for drinking purpose \\
\hline
\end{tabular}

\subsection{Pearson Correlation Analysis of Different Physico-Chemical Parameters of Ropar Wetland}

The Pearson's (r) correlation analysis revealed a positive as well as negative correlation between various physico-chemical parameters at all the four representative sites (S1, S2, S3 and S4) during the present investigations of Ropar wetland from October 2015 to September 2017 and has been presented in Table 7,8,9,10. At all the sites DO and Free $\mathrm{CO}_{2}$ showed significant negative correlation with all other physico-chemical parameters. The rest of the physicochemical parameters i.e. Air Temperature $\left({ }^{\circ} \mathrm{C}\right)$, Water temperature $\left({ }^{\circ} \mathrm{C}\right)$, Conductivity $(\mu \mathrm{S} / \mathrm{cm}), \operatorname{TDS}(\mathrm{mg} / \mathrm{l})$, Turbidity(NTU), $\quad \mathrm{pH}, \quad$ Alkalinity(mg/l), Chlorides(mg/l), Total Hardness(mg/l), Ca++Hardness(mg/l), Mg++Hardness(mg/l), Phosphates(mg/l), Sulphates(mg/l), Nitrates(mg/l), Nitrites(mg/l), Silicates(mg/l) showed positive correlation with each other at all the sites. 
Table 7. Pearson's correlation matrix among physico-chemical parameters at S1 site of Ropar Wetland

\begin{tabular}{|c|c|c|c|c|c|c|c|c|c|c|c|c|c|c|c|c|c|c|}
\hline Parameters & 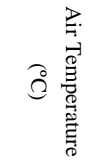 & ○ & 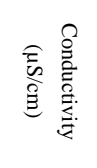 & 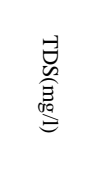 & 晃套 & 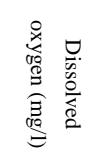 & 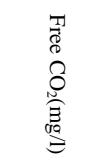 & 蒫 & 丞 & 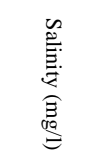 & 付 &  & 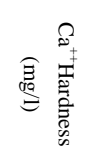 & 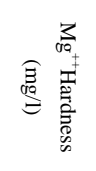 & 高吉 & 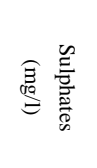 &  &  \\
\hline $\begin{array}{c}\text { Air } \\
\text { Temperature } \\
\left({ }^{\circ} \mathrm{C}\right)\end{array}$ & 0 & & & & & & & & & & & & & & & & & \\
\hline $\begin{array}{c}\text { Water } \\
\text { temperature } \\
\left({ }^{\circ} \mathrm{C}\right) \\
\end{array}$ & 0.92535 & 0 & & & & & & & & & & & & & & & & \\
\hline $\begin{array}{c}\text { Conductivity } \\
(\mu \mathrm{S} / \mathrm{cm})\end{array}$ & 0.7789 & 0.64514 & 0 & & & & & & & & & & & & & & & \\
\hline TDS (mg/l) & 0.69138 & 0.56768 & 0.91247 & 0 & & & & & & & & & & & & & & \\
\hline $\begin{array}{c}\text { Turbidity } \\
\text { (NTU) }\end{array}$ & 0.66342 & 0.49096 & 0.8492 & 0.8306 & 0 & & & & & & & & & & & & & \\
\hline $\begin{array}{c}\text { Dissolved } \\
\text { oxygen (mg/l) }\end{array}$ & -0.90854 & -0.79792 & -0.74618 & -0.69131 & -0.63929 & 0 & & & & & & & & & & & & \\
\hline $\begin{array}{c}\text { Free } \mathrm{CO}_{2} \\
(\mathrm{mg} / \mathrm{l})\end{array}$ & -0.76746 & -0.75104 & -0.7056 & -0.57317 & -0.55168 & 0.51903 & 0 & & & & & & & & & & & \\
\hline $\mathrm{pH}$ & 0.70034 & 0.54977 & 0.81504 & 0.79193 & 0.83062 & -0.71553 & -0.49193 & 0 & & & & & & & & & & \\
\hline $\begin{array}{c}\text { Alkalinity } \\
(\mathrm{mg} / \mathrm{l})\end{array}$ & 0.70649 & 0.60381 & 0.8269 & 0.78934 & 0.85213 & -0.74031 & -0.46833 & 0.87058 & 0 & & & & & & & & & \\
\hline Salinity (mg/l) & 0.68067 & 0.51744 & 0.86613 & 0.83028 & 0.84688 & -0.71852 & -0.4453 & 0.84755 & 0.92935 & 0 & & & & & & & & \\
\hline $\begin{array}{c}\text { Chlorides } \\
(\mathrm{mg} / \mathrm{l})\end{array}$ & 0.66115 & 0.54226 & 0.89912 & 0.87858 & 0.90344 & -0.69078 & -0.51356 & 0.8344 & 0.90548 & 0.91291 & 0 & & & & & & & \\
\hline $\begin{array}{c}\text { Total } \\
\text { Hardness } \\
(\mathrm{mg} / \mathrm{l}) \\
\end{array}$ & 0.76157 & 0.651 & 0.94309 & 0.91459 & 0.86431 & -0.77929 & -0.57829 & 0.83752 & 0.90758 & 0.88265 & 0.94682 & 0 & & & & & & \\
\hline $\begin{array}{c}\mathrm{Ca}^{++} \mathrm{Hardness} \\
(\mathrm{mg} / \mathrm{l})\end{array}$ & 0.46523 & 0.31524 & 0.86833 & 0.81821 & 0.78043 & -0.49326 & -0.47421 & 0.73417 & 0.72288 & 0.79081 & 0.88439 & 0.83608 & 0 & & & & & \\
\hline $\begin{array}{c}\mathrm{Mg}^{++} \text {Hardness } \\
(\mathrm{mg} / \mathrm{l})\end{array}$ & 0.73341 & 0.59418 & 0.86383 & 0.79847 & 0.79431 & -0.78948 & -0.4722 & 0.85063 & 0.93837 & 0.94782 & 0.8837 & 0.88749 & 0.76213 & 0 & & & & \\
\hline $\begin{array}{c}\text { Phosphates } \\
(\mathrm{mg} / \mathrm{l})\end{array}$ & 0.50814 & 0.30638 & 0.64047 & 0.62035 & 0.88111 & -0.57617 & -0.25867 & 0.71467 & 0.76606 & 0.73686 & 0.81219 & 0.73631 & 0.67694 & 0.69471 & 0 & & & \\
\hline $\begin{array}{l}\text { Sulphates } \\
(\mathrm{mg} / \mathrm{l})\end{array}$ & 0.63254 & 0.4444 & 0.6154 & 0.55253 & 0.8335 & -0.67084 & -0.36155 & 0.7301 & 0.74603 & 0.69157 & 0.75286 & 0.6912 & 0.59933 & 0.69849 & 0.95458 & 0 & & \\
\hline Nitrates (mg/l) & 0.44745 & 0.27353 & 0.7595 & 0.77906 & 0.90041 & -0.47097 & -0.34898 & 0.69348 & 0.69639 & 0.73742 & 0.84175 & 0.77245 & 0.84279 & 0.63733 & 0.86388 & 0.75471 & 0 & \\
\hline Nitrites (mg/l) & 0.3891 & 0.23428 & 0.68813 & 0.72528 & 0.79505 & -0.39291 & -0.34338 & 0.60093 & 0.50586 & 0.56924 & 0.72656 & 0.66854 & 0.77538 & 0.47372 & 0.74156 & 0.64182 & 0.93034 & 0 \\
\hline $\begin{array}{c}\text { Silicates } \\
(\mathrm{mg} / \mathrm{l})\end{array}$ & 0.61136 & 0.4276 & 0.63852 & 0.57721 & 0.84788 & -0.57223 & -0.46003 & 0.70505 & 0.63953 & 0.61653 & 0.70704 & 0.63744 & 0.61029 & 0.59029 & 0.88737 & 0.92077 & 0.80557 & 0.79143 \\
\hline
\end{tabular}


Table 8. Pearson's correlation matrix among physico-chemical parameters at S2 site of Ropar Wetland

\begin{tabular}{|c|c|c|c|c|c|c|c|c|c|c|c|c|c|c|c|c|c|c|}
\hline Parameters & 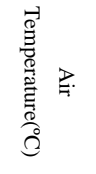 & 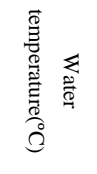 &  & 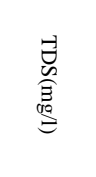 & 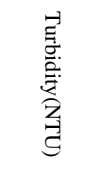 & 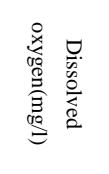 & 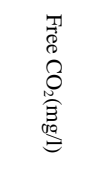 & T & 突 & 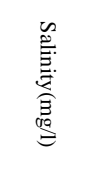 & 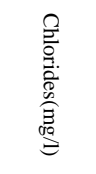 & 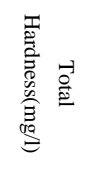 & 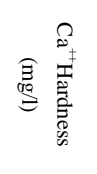 & 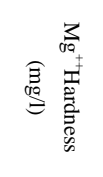 &  & 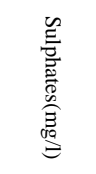 & 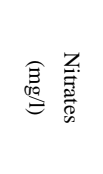 & 喜杰: \\
\hline $\begin{array}{c}\text { Air } \\
\text { Temperature } \\
\left({ }^{\circ} \mathrm{C}\right) \\
\end{array}$ & 0 & & & & & & & & & & & & & & & & & \\
\hline $\begin{array}{c}\text { Water } \\
\text { temperature } \\
\left({ }^{\circ} \mathrm{C}\right) \\
\end{array}$ & 0.98579 & 0 & & & & & & & & & & & & & & & & \\
\hline $\begin{array}{c}\text { Conductivity } \\
(\mu \mathrm{S} / \mathrm{cm})\end{array}$ & 0.92253 & 0.88301 & 0 & & & & & & & & & & & & & & & \\
\hline TDS (mg/l) & 0.71823 & 0.73489 & 0.79467 & 0 & & & & & & & & & & & & & & \\
\hline $\begin{array}{c}\text { Turbidity } \\
\text { (NTU) }\end{array}$ & 0.87094 & 0.86123 & 0.87292 & 0.83345 & 0 & & & & & & & & & & & & & \\
\hline $\begin{array}{c}\text { Dissolved } \\
\text { oxygen (mg/l) }\end{array}$ & -0.78356 & -0.76738 & -0.82279 & -0.75558 & -0.86183 & 0 & & & & & & & & & & & & \\
\hline $\begin{array}{c}\text { Free } \mathrm{CO}_{2} \\
(\mathrm{mg} / \mathrm{l})\end{array}$ & -0.76146 & -0.75593 & -0.65633 & -0.52851 & -0.64861 & 0.51525 & 0 & & & & & & & & & & & \\
\hline $\mathrm{pH}$ & 0.78868 & 0.78116 & 0.83322 & 0.88442 & 0.87382 & -0.72228 & $\begin{array}{l}-0.70869 \\
\end{array}$ & 0 & & & & & & & & & & \\
\hline $\begin{array}{c}\text { Alkalinity } \\
(\mathrm{mg} / \mathrm{l})\end{array}$ & 0.82755 & 0.80983 & 0.85713 & 0.79478 & 0.94564 & -0.90682 & -0.5354 & 0.78159 & 0 & & & & & & & & & \\
\hline Salinity (mg/l) & 0.63514 & 0.65736 & 0.65289 & 0.81841 & 0.84923 & -0.66668 & -0.44963 & 0.83162 & 0.82108 & 0 & & & & & & & & \\
\hline $\begin{array}{c}\text { Chlorides } \\
(\mathrm{mg} / \mathrm{l})\end{array}$ & 0.45466 & 0.4457 & 0.58254 & 0.80751 & 0.70467 & -0.7122 & -0.38508 & 0.75822 & 0.75906 & 0.78814 & 0 & & & & & & & \\
\hline $\begin{array}{c}\text { Total } \\
\text { Hardness } \\
(\mathrm{mg} / \mathrm{l})\end{array}$ & 0.57498 & 0.58024 & 0.68282 & 0.82545 & 0.75164 & -0.74142 & -0.28616 & 0.67941 & 0.82508 & 0.78725 & 0.80589 & 0 & & & & & & \\
\hline $\begin{array}{c}\mathrm{Ca}^{++} \text {Hardness } \\
(\mathrm{mg} / \mathrm{l})\end{array}$ & 0.46596 & 0.45667 & 0.54571 & 0.70463 & 0.6691 & -0.59677 & -0.40416 & 0.76835 & 0.66544 & 0.866 & 0.78792 & 0.73754 & 0 & & & & & \\
\hline $\begin{array}{l}\mathrm{Mg}^{++} \text {Hardness } \\
(\mathrm{mg} / \mathrm{l})\end{array}$ & 0.60991 & 0.61689 & 0.58484 & 0.58797 & 0.77148 & -0.67212 & -0.33386 & 0.5604 & 0.80596 & 0.76334 & 0.54382 & 0.69308 & 0.58242 & 0 & & & & \\
\hline $\begin{array}{l}\text { Phosphates } \\
\text { (mg/l) }\end{array}$ & 0.73611 & 0.73652 & 0.82194 & 0.88471 & 0.89708 & -0.89876 & -0.55937 & 0.865 & 0.91332 & 0.81484 & 0.85029 & 0.81153 & 0.71196 & 0.63147 & 0 & & & \\
\hline $\begin{array}{c}\text { Sulphates } \\
(\mathrm{mg} / \mathrm{l})\end{array}$ & 0.86063 & 0.85787 & 0.89303 & 0.89464 & 0.89733 & -0.8629 & -0.6149 & 0.88489 & 0.85838 & 0.70338 & 0.69748 & 0.69713 & 0.55172 & 0.56462 & 0.90341 & 0 & & \\
\hline Nitrates (mg/l) & 0.50786 & 0.53292 & 0.59517 & 0.8879 & 0.70068 & -0.71515 & -0.42543 & 0.78634 & 0.67482 & 0.7316 & 0.85733 & 0.72219 & 0.698 & 0.40829 & 0.8645 & 0.79962 & 0 & \\
\hline Nitrites (mg/l) & 0.27774 & 0.29497 & 0.41777 & 0.76219 & 0.4997 & -0.56284 & -0.2739 & 0.63981 & 0.47938 & 0.56757 & 0.81925 & 0.56014 & 0.58759 & 0.24911 & 0.72418 & 0.64839 & 0.90699 & 0 \\
\hline $\begin{array}{c}\text { Silicates } \\
(\mathrm{mg} / \mathrm{l})\end{array}$ & 0.61173 & 0.59152 & 0.66872 & 0.70282 & 0.79542 & -0.79232 & -0.67436 & 0.82926 & 0.76039 & 0.75189 & 0.79997 & 0.59245 & 0.75646 & 0.56172 & 0.85897 & 0.72529 & 0.75949 & 0.64871 \\
\hline
\end{tabular}


Table 9. Pearson’s correlation matrix among physico-chemical parameters at S3 site of Ropar Wetland

\begin{tabular}{|c|c|c|c|c|c|c|c|c|c|c|c|c|c|c|c|c|c|c|}
\hline Parameters & 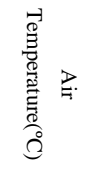 & 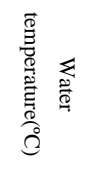 & 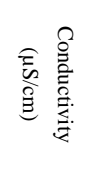 & 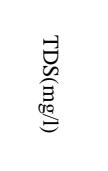 &  &  & 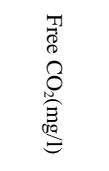 & T: & 突 & 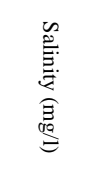 &  &  & 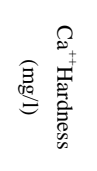 & 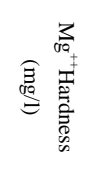 & 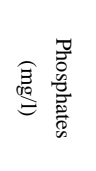 & 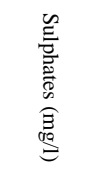 & 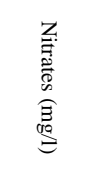 &  \\
\hline $\begin{array}{c}\text { Air } \\
\text { Temperature } \\
\left({ }^{\circ} \mathrm{C}\right)\end{array}$ & 0 & & & & & & & & & & & & & & & & & \\
\hline $\begin{array}{c}\text { Water } \\
\text { temperature } \\
\left({ }^{\circ} \mathrm{C}\right)\end{array}$ & 0.97606 & 0 & & & & & & & & & & & & & & & & \\
\hline $\begin{array}{c}\text { Conductivity } \\
(\mu \mathrm{S} / \mathrm{cm})\end{array}$ & 0.77592 & 0.77472 & 0 & & & & & & & & & & & & & & & \\
\hline TDS (mg/l) & 0.61573 & 0.62425 & 0.74925 & 0 & & & & & & & & & & & & & & \\
\hline $\begin{array}{c}\text { Turbidity } \\
\text { (NTU) }\end{array}$ & 0.6191 & 0.58989 & 0.66962 & 0.20696 & 0 & & & & & & & & & & & & & \\
\hline $\begin{array}{c}\text { Dissolved } \\
\text { oxygen }(\mathrm{mg} / \mathrm{l})\end{array}$ & -0.85743 & -0.84351 & -0.73453 & -0.65649 & -0.56746 & 0 & & & & & & & & & & & & \\
\hline $\begin{array}{c}\text { Free } \mathrm{CO}_{2} \\
(\mathrm{mg} / \mathrm{l})\end{array}$ & -0.79369 & -0.80445 & -0.87948 & -0.56024 & -0.72487 & 0.72687 & 0 & & & & & & & & & & & \\
\hline $\mathrm{pH}$ & 0.7116 & 0.68216 & 0.89133 & 0.74103 & 0.67025 & -0.66319 & -0.80313 & 0 & & & & & & & & & & \\
\hline $\begin{array}{c}\begin{array}{c}\text { Alkalinity } \\
(\mathrm{mg} / \mathrm{l})\end{array} \\
\end{array}$ & 0.6282 & 0.6597 & 0.86125 & 0.80127 & 0.54926 & -0.70511 & -0.7461 & 0.79692 & 0 & & & & & & & & & \\
\hline Salinity (mg/l) & 0.6479 & 0.63478 & 0.82444 & 0.76791 & 0.47691 & -0.78048 & -0.73399 & 0.75229 & 0.89188 & 0 & & & & & & & & \\
\hline $\begin{array}{c}\text { Chlorides } \\
(\mathrm{mg} / \mathrm{l})\end{array}$ & 0.70772 & 0.7122 & 0.94791 & 0.73099 & 0.64194 & -0.73975 & -0.85299 & 0.88337 & 0.88649 & 0.83439 & 0 & & & & & & & \\
\hline $\begin{array}{c}\text { Total } \\
\text { Hardness } \\
(\mathrm{mg} / \mathrm{l})\end{array}$ & 0.7154 & 0.72038 & 0.92348 & 0.79356 & 0.62517 & -0.80657 & -0.80001 & 0.84382 & 0.88639 & 0.84659 & 0.95265 & 0 & & & & & & \\
\hline $\begin{array}{c}\mathrm{Ca}^{++} \mathrm{Hardness} \\
(\mathrm{mg} / \mathrm{l})\end{array}$ & 0.53329 & 0.56083 & 0.84704 & 0.8442 & 0.38165 & -0.56138 & -0.66326 & 0.80394 & 0.84 & 0.77349 & 0.85585 & 0.8435 & 0 & & & & & \\
\hline $\begin{array}{c}\mathrm{Mg}^{++} \text {Hardness } \\
(\mathrm{mg} / \mathrm{l})\end{array}$ & 0.69994 & 0.69372 & 0.86245 & 0.7886 & 0.53848 & -0.80668 & -0.78843 & 0.81796 & 0.92599 & 0.9399 & 0.8602 & 0.87787 & 0.78004 & 0 & & & & \\
\hline $\begin{array}{c}\text { Phosphates } \\
\text { (mg/l) }\end{array}$ & 0.58598 & 0.59439 & 0.83132 & 0.43794 & 0.84859 & -0.60592 & -0.78947 & 0.7465 & 0.76873 & 0.70868 & 0.85023 & 0.82674 & 0.67961 & 0.73532 & 0 & & & \\
\hline $\begin{array}{c}\text { Sulphates } \\
(\mathrm{mg} / \mathrm{l})\end{array}$ & 0.42993 & 0.40242 & 0.50785 & 0.018558 & 0.84564 & -0.45186 & -0.64371 & 0.51385 & 0.34057 & 0.38896 & 0.52962 & 0.4834 & 0.25509 & 0.40213 & 0.73917 & 0 & & \\
\hline Nitrates (mg/l) & 0.33608 & 0.32055 & 0.71871 & 0.36721 & 0.65642 & -0.38532 & -0.63422 & 0.6783 & 0.60884 & 0.62306 & 0.77897 & 0.7004 & 0.67639 & 0.55117 & 0.80573 & 0.73963 & 0 & \\
\hline Nitrites (mg/l) & 0.33463 & 0.31943 & 0.66586 & 0.38598 & 0.49476 & -0.37387 & -0.52084 & 0.64032 & 0.48327 & 0.53733 & 0.72255 & 0.64304 & 0.69991 & 0.45333 & 0.68464 & 0.61577 & 0.92236 & 0 \\
\hline $\begin{array}{c}\text { Silicates } \\
(\mathrm{mg} / \mathrm{l})\end{array}$ & 0.60953 & 0.59821 & 0.8485 & 0.63669 & 0.6273 & -0.59531 & -0.78068 & 0.86955 & 0.66803 & 0.68419 & 0.86136 & 0.81044 & 0.82675 & 0.68783 & 0.76268 & 0.65199 & 0.85537 & 0.883 \\
\hline
\end{tabular}


Table 10. Pearson's correlation matrix among physico-chemical parameters at S4 site of Ropar Wetland

\begin{tabular}{|c|c|c|c|c|c|c|c|c|c|c|c|c|c|c|c|c|c|c|}
\hline Parameters & 兽 & 仓 & 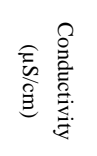 & 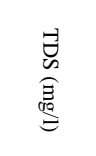 & 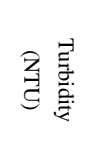 & 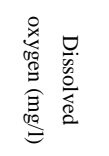 & 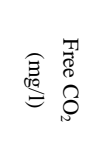 & T: & 営 & 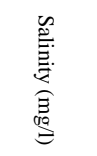 & 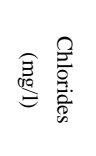 & 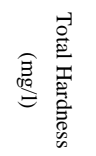 & 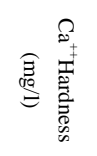 & 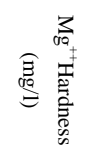 & 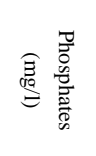 &  & 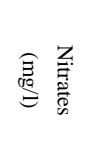 & 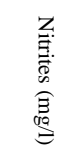 \\
\hline $\begin{array}{c}\text { Air } \\
\text { Temperature } \\
\left({ }^{\circ} \mathrm{C}\right) \\
\end{array}$ & 0 & & & & & & & & & & & & & & & & & \\
\hline $\begin{array}{c}\text { Water } \\
\text { temperature } \\
\left({ }^{\circ} \mathrm{C}\right)\end{array}$ & 0.77396 & 0 & & & & & & & & & & & & & & & & \\
\hline $\begin{array}{c}\text { Conductivity } \\
(\mu \mathrm{S} / \mathrm{cm})\end{array}$ & 0.85837 & 0.80788 & 0 & & & & & & & & & & & & & & & \\
\hline TDS (mg/l) & 0.62824 & 0.70863 & 0.81496 & 0 & & & & & & & & & & & & & & \\
\hline $\begin{array}{l}\text { Turbidity } \\
\text { (NTU) }\end{array}$ & 0.71017 & 0.68082 & 0.88802 & 0.8562 & 0 & & & & & & & & & & & & & \\
\hline $\begin{array}{c}\text { Dissolved } \\
\text { oxygen (mg/l) }\end{array}$ & -0.72634 & -0.71395 & -0.88979 & -0.77089 & -0.90687 & 0 & & & & & & & & & & & & \\
\hline $\begin{array}{c}\text { Free } \mathrm{CO}_{2} \\
(\mathrm{mg} / \mathrm{l})\end{array}$ & -0.68236 & -0.63176 & -0.61715 & -0.52557 & -0.48404 & 0.36295 & 0 & & & & & & & & & & & \\
\hline $\mathrm{pH}$ & 0.79973 & 0.6507 & 0.82321 & 0.74442 & 0.80053 & -0.73774 & -0.61822 & 0 & & & & & & & & & & \\
\hline $\begin{array}{l}\text { Alkalinity } \\
(\mathrm{mg} / \mathrm{l})\end{array}$ & 0.7077 & 0.63952 & 0.8292 & 0.74899 & 0.94302 & -0.88595 & -0.41923 & 0.7082 & 0 & & & & & & & & & \\
\hline Salinity (mg/l) & 0.60486 & 0.58406 & 0.72638 & 0.891 & 0.85687 & -0.71319 & -0.35647 & 0.67366 & 0.82674 & 0 & & & & & & & & \\
\hline $\begin{array}{l}\text { Chlorides } \\
(\mathrm{mg} / \mathrm{l})\end{array}$ & 0.55693 & 0.57322 & 0.75699 & 0.80695 & 0.91017 & -0.81433 & -0.35953 & 0.8157 & 0.85379 & 0.83441 & 0 & & & & & & & \\
\hline $\begin{array}{c}\text { Total } \\
\text { Hardness } \\
(\mathrm{mg} / \mathrm{l})\end{array}$ & 0.63331 & 0.64931 & 0.82455 & 0.85538 & 0.88135 & -0.7919 & -0.42249 & 0.77441 & 0.83327 & 0.88381 & 0.88487 & 0 & & & & & & \\
\hline $\begin{array}{c}\mathrm{Ca}^{++} \mathrm{Hardness} \\
(\mathrm{mg} / \mathrm{l})\end{array}$ & 0.38408 & 0.42805 & 0.62639 & 0.83131 & 0.70886 & -0.5356 & -0.30257 & 0.62634 & 0.59917 & 0.86094 & 0.74846 & 0.82889 & 0 & & & & & \\
\hline $\begin{array}{c}\mathrm{Mg}^{++} \text {Hardness } \\
(\mathrm{mg} / \mathrm{l})\end{array}$ & 0.56301 & 0.65593 & 0.69688 & 0.70859 & 0.79475 & -0.66988 & -0.31017 & 0.52043 & 0.84305 & 0.82337 & 0.69909 & 0.81279 & 0.68291 & 0 & & & & \\
\hline $\begin{array}{c}\text { Phosphates } \\
\text { (mg/l) }\end{array}$ & 0.6635 & 0.70998 & 0.85006 & 0.84918 & 0.91965 & -0.88421 & -0.43015 & 0.84346 & 0.85191 & 0.81741 & 0.93666 & 0.91624 & 0.72504 & 0.7159 & 0 & & & \\
\hline $\begin{array}{c}\text { Sulphates } \\
(\mathrm{mg} / \mathrm{l})\end{array}$ & 0.63268 & 0.56282 & 0.8057 & 0.71326 & 0.87151 & -0.8459 & -0.41107 & 0.88062 & 0.79853 & 0.6894 & 0.88091 & 0.7858 & 0.60771 & 0.56427 & 0.8846 & 0 & & \\
\hline Nitrates (mg/l) & 0.24859 & 0.29759 & 0.50477 & 0.69025 & 0.56114 & -0.51432 & -0.30917 & 0.64987 & 0.36778 & 0.56401 & 0.68233 & 0.66327 & 0.71781 & 0.2471 & 0.71218 & 0.69705 & 0 & \\
\hline Nitrites (mg/l) & 0.16477 & 0.22202 & 0.45777 & 0.66438 & 0.63208 & -0.55242 & -0.1545 & 0.55669 & 0.47694 & 0.62914 & 0.73851 & 0.65462 & 0.70803 & 0.35204 & 0.73352 & 0.73381 & 0.90597 & 0 \\
\hline $\begin{array}{c}\text { Silicates } \\
(\mathrm{mg} / \mathrm{l})\end{array}$ & 0.58037 & 0.54475 & 0.78255 & 0.81204 & 0.86765 & -0.80084 & -0.48236 & 0.8786 & 0.76289 & 0.75143 & 0.89775 & 0.85291 & 0.74912 & 0.5843 & 0.92612 & 0.9268 & 0.85016 & 0.83424 \\
\hline
\end{tabular}




\subsection{Water Quality Assessment of Ropar Wetland on the Basis of Water Quality Index (WQI)}

WQI can be defined as the index which reflects the combinatorial effect of various water quality parameters and provides a single number which gives information about the overall water quality of studied water body. Water quality degradation has adversely disturbed ecosystem balance and contaminated various ground and surface water resources. To ascertain the quality of water for public usage and other purposes, we calculated water quality index (WQI) of Ropar wetland at four representative sites (S1, S2, S3 and S4). We determined WQI on the basis of various physico-chemical parameters like TDS, Dissolved oxygen, pH, Alkalinity, Salinity, Chlorides, Total Hardness, $\mathrm{Ca}++$ Hardness, $\mathrm{Mg}++$ Hardness and sulphates at all the sites. It was found in present study that various physico-chemical parameters greatly influence water quality of under study wetland. The average results of these parameters were compared with National and International standards for drinking water quality viz. Indian Standard Institute (ISI, 1973), Indian Council of Medical Research (ICMR, 1975) and World Health Organization (WHO, 1992) and are represented in Table 5, all parameters come under permissible limits of drinking water standards except for alkalinity, $\mathrm{pH}$, magnesium hardness. The WQI values of Ropar wetland at all the sites (S1, S2, S3 and S4) was observed to be 76.92, 73.93, 76.17 and 73.35 and are depicted in Table 1,2,3,4. It could be inferred that water quality at Ropar wetland is "Poor" at S2 site and S4 site and "Very Poor" at S1 site and S3 site according to the values given by (Chatterji and Raziuddin, 2002) shown in Table 6. WQI for the under study wetland depicted its very poor water quality, hence unsafe for human usage. This can be attributed to the presence of high level pollutants, industrial effluents from National Fertilizer Limit (NFL) Nangal, ash from thermal plant and silt from cement plant and catchment area, domestic sewage and surface run-off from agricultural fields in Ropar wetland. Thus it can be inferred that if not dealt on time, this poor water quality of Ropar wetland can have deleterious impact on its ecosystem.

\section{Discussion}

To sustain natural ecosystem and human development water resources play a pivotal role. The physico-chemical and biological characteristics depict the state of an aquatic ecosystem and their abundance corresponds to the good quality of water. Water is an essential component of the environment and it sustains life on the earth. All organisms depend on water for their survival (Smitha et al., 2007). In order to identify magnitude and source of any pollution load it is important to monitor these parameters. The water quality and correlation between different physico-chemical parameters were analyzed statistically by using Pearson correlation analysis at all sites of Ropar wetland, Punjab, India. During the present investigations $\mathrm{DO}$ and Free $\mathrm{CO}_{2}$ showed significant negative correlation with all other physico-chemical parameters at all the sites. Our results are in conformity with Venkatesharajuet al. (2010) who also analyzed a negative correlation of DO with turbidity and COD. The reason behind this decreased value of DO in water is the presence of organic and inorganic compounds (Agarwal and Agarwal, 2013). Our results are again supported by a number of researchers who evidenced similar observations during their study period (Joshi et al., 2009; Khatoon et al., 2013 and Abir, 2014; Ismail (2007). Total Dissolved Solids during the study period showed significant positive relationship with $\mathrm{Ca}^{++}$, salinity and Total Hardness and significant negative relationship with DO. Comparable findings were observed by Bhandari and Nayal, 2008 and Sen et al., 2011. Total Hardness during the study period shows significant positive relationship with $\mathrm{Mg}^{++}, \mathrm{Cl}^{-}, \mathrm{Ca}++$ and significant negative relationship with DO. Our results are in concordance with Bhandari and Nayal, 2008; Joshi et al., 2009; Khatoon et al., 2013 and Abir, 2014. Chloride shows significant positive relationship with $\mathrm{Ca}++, \mathrm{Mg}^{++}$, salinity, Total Hardness and significant negative relationship with DO. Our results are similar to those observed by Bhandari and Nayal, 2008; Khatoon et al., 2013; Brraich and Kaur, 2015. EC showed significant positive correlation with turbidity, TDS, Total Hardness whereas EC showed negative correlation with DO. Our results are analogous to Khatoon et al., 2013; Prasad et al., 2014 and Abir, 2014. During the study period $\mathrm{pH}$ showed significant negative relationship with Free Carbon dioxide and DO. Present results are supported by Sen et al., 2011; Khatoon et al., 2013; Qureshimatvaet al., 2015; Brraich and Kaur 2015. Water temperature shows significant positive relationship with TDS, Electrical conductivity, alkalinity, Chloride and significant negative relationship with DO which is in accordance with the study of Brraich and Kaur, 2015 they worked on nangal wetland, punjab (India) by taking assessment of physico-chemical parameters and water quality index.

During the present study water quality of Ropar wetland was found to be greatly affected by various physico-chemical parameters. The average results of these parameters were compared with National and International standards for drinking water quality viz. Indian Standard Institute (ISI, 1973), Indian Council of Medical Research (ICMR, 1975) and World Health Organization (WHO, 1992). All parameters come under permissible limits of drinking water standards except for alkalinity, $\mathrm{pH}$ and magnesium hardness. Based on WQI and on comparing the water quality parameters with national and international standards, it could be inferred that water quality at S2 and S4 sites is "poor" and at S1 and S3 sites is very poor. This can be attributed to the presence of high level pollutants, industrial effluents, domestic sewage and surface run-off from agricultural fields. Thus it can be inferred that if not 
dealt on time, this poor water quality of Ropar wetland can have deleterious impact on its ecosystem. These values were found to be higher than reported by other workers. Present results are contradictory to a study carried out on Uyyakondan channel of river Cauvery at Tiruchirapalli and Kabini river in Karnataka bye Jameel and Hussain, 2005 and Padmanabha and Belagali, 2007 respectively.

\section{Conclusions}

Various natural processes occurring during the rainy seasons, anthropogenic influences from domestic wastewater, agricultural activities, industrial effluents from National Fertilizer Limit (NFL) Nangal, ash from thermal plant and silt from cement plant and catchment area affect the water quality of Ropar wetland. The fluctuation in physico-chemical characteristics have also been observed during the present course of work. WQI for the under study wetland depicted its poor water quality as such is unsafe for human usage. Thus it can be inferred that this poor water quality of Ropar wetland can have deleterious impact on its ecosystem if left unattended and not dealt well on time. At this moment, preservation of the wetlands from the anthropogenic threats is one of the key concerns and this can be achieved by creating awareness regarding about the importance of wetlands among the people by applying appropriate communication strategy.

\section{REFERENCES}

[1] Abir S. 2014. Seasonal Variations in Physico-Chemical Characteristics of Rudrasagar Wetland - A Ramsar Site, Tripura, North East, India. Res. J. Chem. Sci., 4(1): 31-40.

[2] Agarwal, M. and Agarwal, A. 2013. Linear regression and correlation analysis of water quality parameters: A case study of river Kosi at district Rampur India. Inter. J. Innovative res. Sci. Eng. Tech. 2(12): 7223-7229.

[3] Ali, M., Salam, A., Azeem, A., Shafique, M. and Khan, B.A. 2000. Studies on the effect of seasonal variations on physical and chemical characteristics of mixed water from Rivers Ravi and Chenab at union site in Pakistan. J. Res. B. Z. Univ. Multan, 2: 1-17.

[4] American Public Health Association 2012. Standard methods for the examination of water and waste water, 21st (Ed.). New York: American Public Health Association, American Water Works Association \& Water Environment Federation.

[5] Bhandari, N.S. and Nayal, K. 2008. Correlation Study on Physico-Chemical Parameters and Quality Assessment of Kosi River Water, Uttarakhand. E J. Chem., 5(2): 342-346.

[6] Bini, L.M., Thomaz, S.M., Murphy, K.J. and Camargo, A.F.M. 1999. Aquatic macrophyte distribution in relation to water and sediment conditions in the Itaipu Reservoir, Brazil. Hydrobiol.,415: 147-154.
[7] Boyd, C.E. and Tucker, C.S. 1998. Pond aquaculture and water quality management. London: Kluwer Academic Pub., pp. 44-48.

[8] Brown, R.M., McCleiland, N.J., Deininger, R.A. and O' Connor, M.F. 1972. A water quality index- crossing the psycho- logical barrier (Ed. Jenkis, S.H.). Proc. Inter. Conf. water Poll. Res., Jerusalem, 6: 787-797.

[9] Brraich, O.S. and Kaur, R. 2015. Assessment of physico-chemical parameters and water quality index of Nangal wetland, Punjab (India). J. Env. Bio-Sci., 29 (1): 33-39.

[10] Chhatawal, G.R. 1998. Encyclopedia of Environmental Biology. New Delhi, India: Anmol Pub. Pvt. Ltd., 2: 287301.

[11] Deininger, R.A. and Maciunas, J.J. 1971. A water quality of environmental and industrial health, school of public health, University of Michigan, Ann Abor, Michigan.

[12] Harkins, R.D. 1974. An objective water quality index. J. water Pollut. Control Fed.,46: 589.

[13] Horton, R.K. 1965. An index number system for rating water quality. Journal-Water Pollution Control Federation. 37: 300-305.

[14] ICMR 1975. In: Manual of standards of quality for drinking water supplies. New Delhi: ICMR.

[15] ISI 1973. In: Indian standard methods for sampling and test (physical and chemical) for water used in industry. Mank Bhawan: Indian Standard Institute.

[16] Ismail, B.S. 2007. A Physico-chemical assessment of the Bebar river, Pahang, Malaysia. Glob. J. Environ. Res., 1: 07-11.

[17] Jameel, A.A. and Hussain, A.Z. 2005. Water quality index of Uyyakondan Channel of river Cauvery at Tiruchirapalli. Indian J. Environ. Prot.,25(10): 941-942.

[18] Jindal, R. and Sharma, C. 2010. Studies on water quality of Sutlej River around Ludhiana with reference to physicochemical parameters. Environ. Monit. Assess., 174: 417-425.

[19] Joshi, D.M., Bhandari, N.S., Kumar, A. and Agrawal, N. 2009. Statistical analysis of physicochemical parameters of water of river ganga in haridwar district. Rasay. J. Chem., 2(3): 579-587.

[20] Khatoon, N., Khan, A.H., Rehman, M. and Pathak, V. 2013. Correlation Study for the Assessment of Water Quality and Its Parameters of Ganga River, Kanpur, Uttar Pradesh, India. J. App. Chem., 5(3): 80-90

[21] Ladhar, S.S. 2005. Ropar Wetland. Chandigarh: Punjab State Council for Science and Technology.

[22] Lawson, E.O. 2011. Physico-chemical Parameters and Heavy Metal Content of Water from Mangrove swamps of Lagos Lagoon, Lagos, Nigeria. Advan. Biol. Res., 5: 08-21.

[23] Lloyd, R. 1992. Pollution and Fresh Water Fish, Fishing News Books.

[24] Lougheed, V.L., Crosbie, B. and Chow-Fraser, P. 2001. Primary determinants of macrophyte community structure 
in 62 marshes across the Great Lakes basin: latitude, land use, and water quality effects. Can. J. Fish. Aquat. Sci.,58: 1603-1612.

[25] Magee, T.K., Ernst, T.L., Kentula, M.E, and Dwire, K.A. 1999. Floristic comparison of freshwater wetlands in an urbanizing environment. Wetlands,19(3): 517-534.

[26] Mishra, P. and Patel, R.K. 2005. Some aspects of the quality of water in and around Rorkela, thesis.

[27] Padmanabha, B. and Belagali, S.L. 2007. Water quality index of Kabini river in the Kallaahally village of Nanjangud taluk, Mysore district, Karnataka. J. Environ. Sci. Eng.,49(1), 48-50.

[28] Prasad, M., Reddy, B.M., Reddy, M.R. and Sunitha, V. 2014. Studies on physicochemical parameters to assess the water quality in Obulavaripalli Mandal of YSR (Kadapa) District, Andhra Pradesh, India. Int. J. Curr. Res. Acad. Revi., 2(12): 31-41.

[29] Qureshimatva, U.M., Maurya, R.R., Gamit, S.B. and Solanki, H.A. 2015. Studies on the Physico Chemical Parameters and Correlation Coefficient of Sarkhej Roza Lake, District Ahmedabad, Gujarat. Ind. J. Environ. Anal. Toxicol., 5(4): 284-288.

[30] Ramakrishnaiah, C.R., Sadashivaiah, C., Ranganna, G., 2009. Assessment of water quality Index for the groundwater in Tumkur Taluk, KarnatakaState, India. Electron. J. Chem., 6(2): 523-530.

[31] Sen, S., Paul, M.K. and Borah, M. 2011. Study of some Physico-Chemical Parameters of Pond and River water with reference to Correlation Study. Int. J. Chem. Tech. Res., 3(4): 1802-1807

[32] Sisodia, R. and Moundiotiya, C. 2006. Assessment of the water quality index of wetland Kalakho Lake, Rajasthan, India. J. Environ. Hydrol.,14: 1-11.

[33] Smitha, P.G., Byrappa, K. and Ramaswamy, S.N. 2007. Physico-chemical characteristics of water samples of Bantwal Taluk, South-Western Karnataka, India. J. Environ. Biol., 28: 591-595.

[34] Toivonen, H. and Huttunen, P. 1995. Aquatic macrophytes and ecological gradients in 57 small lakes in southern Finland. Aquat. Bot., 51: 197-221.

[35] Trivedi, R.K. and Goel, P.K. 1984. Chemical and Biological Methods for Water Pollution Studies. India: Environmental Publications.

[36] Venkatesharaju, K., Ravikumar, P., Someshekar, P.K. and Prakash, K. L. 2010. Physico chemical and bacteriological investigation on the river Couvery of Kollegal stretch in Karnataka: Kathmandu. Uni. J. of Sci., Eng. And Tech. 6(1): 50-59.

[37] W.H.O. 1992. In: International standards for drinking water. Geneva: World Health Organisation. 